\title{
EFEITO DA CONCENTRAÇÃO MOLAR TOTAL NA CINÉTICA DE ADSORÇÃO BICOMPOSTO DE ÍONS PRATA E COBRE EM ARGILA BENTONÍTICA
}

\author{
E. D. FREITAS ${ }^{1}$, A. C. R. CARMO ${ }^{1}$ A. F. ALMEIDA NETO ${ }^{1}$, M. G. A. VIEIRA $^{1 *}$ \\ ${ }^{1}$ Universidade Estadual de Campinas, Faculdade de Engenharia Química \\ e-mail: melissagav@feq.unicamp.br
}

\begin{abstract}
RESUMO
A remoção de compostos inorgânicos de efluentes industriais tem ganhado bastante destaque, especialmente no que se refere aos metais tóxicos. Compostos solúveis em água e bioacumulativos, esses metais podem trazer sérios danos ao meio ambiente e aos seres vivos, o que tem gerado legislações cada vez mais restritas à emissão desses contaminantes nos corpos d'água. Diversos métodos podem ser empregados em sua remoção, sendo o mais comum a precipitação química. No entanto, inflexibilidade no processo ou falta de ineficiência nos métodos convencionais, vem reforçando o emprego de novas técnicas, com destaque ao processo de adsorção, que tem apresentado bons resultados, não só em eficiência, mas também econômicos. No presente trabalho foi avaliado o emprego de argila bentonítica Verde-lodo para remoção de cobre e prata, em soluções equimolares, porém com concentrações diferentes. Esse adsorvente se mostrou eficiente na remoção monocomposta de ambos os metais. Como resultado, obteve-se maior adsorção de cobre, quando na presença de prata, a qual aparentou ter sofrido inibição, reduzindo sua quantidade adsorvida em sistema bicomposto. Além disso, os modelos cinéticos de pseudoprimeira e pseudossegunda ordem, de difusão intrapartícula e de Boyd foram ajustados aos dados experimentais, fornecendo ajustes razoáveis.
\end{abstract}

\section{INTRODUÇÃO}

A questão da poluição de recursos naturais tem sido amplamente divulgada $\mathrm{e}$ avaliada ao redor do mundo. Nesse contexto, merece destaque a poluição aquática, uma vez que esse é um recurso finito e, muitas vezes, empregado de forma desordenada. Não são raros os casos de indústrias que retiram esse bem do meio ambiente para uso no processo, mas o devolvem contaminado, seja por ineficiência na etapa de tratamento, ou até mesmo a ausência dessa etapa.

Dentre os poluentes, os metais tóxicos são de suma importância, especialmente por sua solubilidade em água e consequente facilidade de absorção por organismos vivos, se acumulando ao longo da cadeia alimentar (BARAKAT, 2011). Particularmente, indústrias que apresentam processos de galvanoplastia se caracterizam pela elevada carga de efluentes contendo esses metais, podendo se destacar entre eles a prata e o cobre (MOREIRA e CARVALHO, 2012).

A prata é um metal precioso e desempenha importantes papéis em muitos aspectos da vida humana, podendo, no entanto, apresentar impactos negativos à saúde do homem e ao ecossistema, mesmo em baixas concentrações. Com a crescente preocupação relativa à sua toxicidade e à escassez de fontes naturais de prata, se torna um desafio não só remover, mas também recuperar esse metal de efluentes aquosos (SONG et al., 2011). Já o cobre é um metal essencial aos organismos vivos, presente em diversas enzimas e proteínas (ALLOWAY, 2010). Em elevadas concentrações, é 
consideravelmente tóxico a seres invertebrados e peixes, fazendo-se necessária também sua remoção de efluentes (HOMEM, 2001).

A remoção desses metais envolve em geral processos físico-químicos de precipitação, troca iônica, extração por solvente, entre outros. No entanto, tais processos não tem se mostrado adequados diante das legislações cada vez mais restritas para despejo de efluentes (AGUIAR e NOVAES, 2002; BARAKAT, 2011). Nesse sentido, o processo de adsorção vem sendo amplamente pesquisado como alternativa econômica e eficiente para remoção de metais tóxicos, mesmo para soluções com baixas concentrações iniciais (YUN et al., 2001).

Estudar o processo de adsorção é, antes de qualquer coisa, escolher um bom sólido adsorvente, capaz de remover a substância de interesse (adsorbato) de forma rápida $\mathrm{e}$ eficiente, com baixo custo e possibilidade de reutilização, além da disponibilidade para eluição das substâncias adsorvidas (BARROS, 2012). O presente trabalho se concentra em avaliar argila Verde-lodo para remoção de prata e cobre em solução binária, uma vez que resultados satisfatórios foram obtidos para solução monocomposta (ALMEIDA NETO, 2011; CANTUARIA, 2014).

Para avaliar a adsorção, pode-se realizar uma análise quantitativa da eficácia de remoção do adsorbato, através da cinética de adsorção. Essa é a primeira etapa para entender os mecanismos envolvidos e fundamentar futuros projetos em maior escala (DING et al., 2012). A cinética depende, entre outros, da interação entre o adsorbato e o adsorvente e das condições do sistema $(\mathrm{pH}$, temperatura), e possibilita obter o tempo de residência necessário ao equilíbrio da remoção (HO, 2004). A avaliação da cinética de adsorção consiste no uso de diversos modelos cinéticos a fim de descrever os mecanismos envolvidos.

\section{MATERIAIS E MÉTODOS}

\subsection{Materiais Utilizados}

A argila bentonítica Verde-lodo, proveniente do estado da Paraíba, Brasil, e fornecida pela Dolomil Ltda, foi moída e peneirada para obtenção de diâmetro médio de 0,855 mm e, em seguida, submetida a um tratamento térmico para calcinação da amostra à $500^{\circ} \mathrm{C}$, por 24 h. Essa etapa é importante para aumentar a estabilidade mecânica e resistência à fluidodinâmica (ALMEIDA NETO, 2011). Como fonte de prata e cobre para as soluções, foram utilizados os sais nitrato de prata e nitrato de cobre, fornecidos pela Merck (Alemanha) e pela Vetec (Brasil), respectivamente.

\subsection{Ensaios de Remoção em Sistema Estático}

Para avaliar a cinética de adsorção de cobre e prata em argila Verde-lodo, empregou-se um sistema de banho finito com agitação magnética constante durante $16 \mathrm{~h}$, para garantir o equilíbrio total. Nesse processo, três soluções foram avaliadas, com a mesma proporção molar de cada íon (50\%), porém em concentrações diferentes, como demonstrado na Tabela 1.

Tabela 1 - Concentrações das soluções empregadas no estudo cinético

\begin{tabular}{ccc}
\hline $\begin{array}{c}\text { Identificação } \\
\text { da Solução }\end{array}$ & $\begin{array}{c}\text { Concentração } \\
\text { de Prata } \\
(\mathrm{mmol} / \mathrm{L})\end{array}$ & $\begin{array}{c}\text { Concentração } \\
\text { de Cobre } \\
(\mathrm{mmol} / \mathrm{L})\end{array}$ \\
\hline $\mathrm{A}$ & 0,5 & 0,5 \\
$\mathrm{~B}$ & 1,0 & 1,0 \\
$\mathrm{C}$ & 2,0 & 2,0 \\
\hline
\end{tabular}

Para o procedimento experimental, 1,0 litro de solução foi colocado em contato com $10 \mathrm{~g}$ de adsorvente, sendo o $\mathrm{pH}$ verificado ao longo de todo o experimento. Em tempos prédeterminados, alíquotas foram retiradas tomando o cuidado de não se ultrapassar o volume total retirado em $8 \%$ do volume 
inicial. Essas alíquotas foram centrifugadas e a concentração dos sobrenadantes, bem como a da solução inicial, determinadas em Espectrofotômetro de Absorção Atômica AAnalyst 100, da PerkinElmer (EUA). A concentração de cada íon na fase sólida no instante $\mathrm{t}$ pode ser determinada em função da concentração obtida no espectrofotômetro $(\mathrm{C}(\mathrm{t}))$, como na Equação 1.

$$
q(t)=\frac{V}{m} .\left(C_{0}-C(t)\right)
$$

\subsection{Modelos cinéticos}

Diversos modelos cinéticos são encontrados na literatura, considerando diferentes fatores determinantes para a velocidade de adsorção.

\subsubsection{Modelo de Pseudoprimeira Ordem}

No modelo de pseudoprimeira ordem, o fator determinante para a velocidade é a resistência à transferência de massa no sólido, e a Equação 2 é empregada para representá-lo (LAGERGREN, 1989).

$\frac{\partial q}{\partial t}=k_{1} \cdot\left(q_{e}-q\right)$

Sabe-se que no tempo inicial $(\mathrm{t}=0)$, a concentração de íons no adsorvente é nula. Resolvendo a Equação 2 segundo esta condição, obtém-se as Equações 3 e 4.

$$
\begin{aligned}
& \ln \left(q_{e}-q\right)=\ln q_{e}-k_{1} \cdot t \\
& q=q_{e} \cdot\left(1-e^{-k_{1} \cdot t}\right)
\end{aligned}
$$

\subsubsection{Modelo de Pseudossegunda Ordem}

O modelo de pseudossegunda ordem foi proposto considerando que o sistema apresenta um comportamento de forças impulsoras não lineares, e pode ser bem descrito pela Equação 5 (HO e McKAY, 1998).

$$
\frac{\partial q}{\partial t}=k_{2} \cdot\left(q_{e}-q\right)^{2}
$$

Mais uma vez, pode-se considerar a concentração inicial no sólido como nula e, integrando a Equação 5, pode-se obter a resolução para esse modelo, exposto na Equação 6.

$$
\frac{q}{q_{e}}=\frac{k_{2} \cdot q_{e} \cdot t}{1+k_{2} \cdot q_{e} \cdot t}
$$

\subsubsection{Modelo de Difusão Intrapartícula}

Nesse modelo, é considerado que a difusão dentro da partícula de sólido ocorre de forma lenta e, por isso, limitante ao processo. Nesse caso, a difusão superficial acontece de forma gradual e linear e o modelo pode ser bem representado pela Equação 7 (WEBER e MORRIS, 1963).

$$
q=k_{i} \cdot t^{1 / 2}
$$

Ao graficar dados de $q$ vs. $t^{1 / 2}$, é possível obter uma reta com inclinação $\mathrm{k}_{\mathrm{i}}$. Entretanto, uma possibilidade é de que ocorra multilinearidade ao plotar esses dados, indicando mais de uma etapa limitante ao processo. Quando isso ocorre, pode-se observar até três etapas distintas. Na primeira, uma adsorção instantânea, ou na superfície externa; em seguida, a adsorção gradual, com difusão intrapartícula limitante; e, finalmente, um equilíbrio final, com difusão intrapartícula reduzindo, devido às menores concentrações de adsorbato na solução. Esse caso pode ser melhor representado pela Equação 8 (CHEN et al., 2003).

$$
q=k_{i} \cdot t^{1 / 2}+c
$$

\subsubsection{Modelo de Boyd}

O Modelo de Boyd (1947) foi proposto com o objetivo de determinar se a etapa limitante é o transporte externo ou a difusão 
intrapartícula. De acordo com esse modelo, a fração de soluto adsorvida em certo tempo $t$ pode ser determinada como na Equação 9.

$$
F=1-\left(\frac{6}{\pi^{2}}\right) \cdot \exp \left(-B_{t}\right)
$$

Sendo:

$$
F=\frac{q}{q_{e}}
$$

$\mathrm{O}$ parâmetro $\mathrm{B}_{\mathrm{t}}$ pode ser obtido ao reescrever a Equação 9 para obter a Equação 11.

$$
B_{t}=-0,4977-\ln (1-F)
$$

Ao graficar $B_{t}$ vs. $t$, pode-se avaliar a linearidade para determinar o mecanismo que controla a adsorção. Caso a reta passe pela origem, a etapa limitante é a difusão intrapartícula; caso contrário, o transporte externo limita o processo. O coeficiente angular dessa reta permite calcular o coeficiente de difusão efetiva (Di), conforme Equação 12 (GUPTA e ALI, 2001).

$$
\alpha=\frac{\pi^{2} \cdot D_{i}}{r^{2}}
$$

\section{RESULTADOS E DISCUSSÃO}

As curvas cinéticas obtidas para as soluções propostas estão apresentadas na Figura 1, com dados de prata e cobre expostos em conjunto. Verifica-se que foi necessário um tempo médio de $300 \mathrm{~min}$ de contato entre a solução e o sólido, para que o equilíbrio fosse atingido. Além disso, constata-se a maior adsorção de cobre em relação à prata, na argila Verde-lodo, em qualquer uma das soluções empregadas. Tal fato condiz com $o$ obtido em sistemas monocompostos de prata e cobre, por adsorção em argila Verde-lodo.
Em ensaios de remoção em sistema estático, Almeida Neto (2011) obteve uma remoção de cobre de $0,085 \mathrm{mmol} / \mathrm{g}$ argila, enquanto Cantuaria (2014) obteve remoção de $0,057 \mathrm{mmol} / \mathrm{g}$ argila para a prata. Quando os metais estão na mesma solução, pode-se observar uma inibição da adsorção de prata na presença de cobre e, consequentemente, sua quantidade adsorvida reduz.

Figura 1 - Curvas cinéticas obtidas para cobre e prata nas Soluções A - C

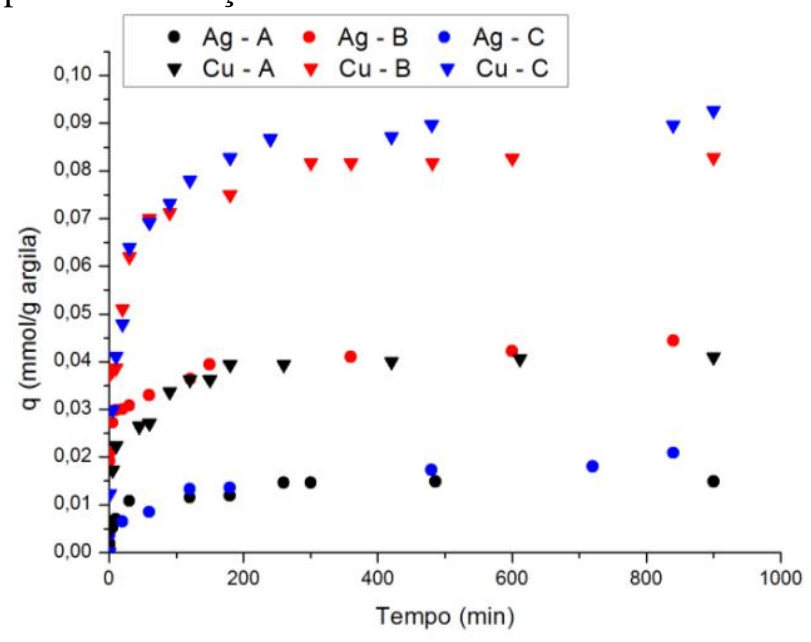

As soluções empregadas são equimolares, variando apenas sua quantidade de íons em solução. Ao se avaliar a influência dessa quantidade de íons na adsorção, pode-se observar o aumento da quantidade adsorvida de prata e cobre em função do aumento da concentração inicial da Solução A para a B. No entanto, no caso da Solução C, com a maior concentração inicial total, esse comportamento não é observado. A quantidade adsorvida de prata reduz em relação à da Solução B, e a de cobre apresenta apenas um pequeno incremento. Isso pode ser explicado pela quantidade total de íons presente em solução. O valor elevado dessa grandeza pode ter colaborado com a redução da adsorção, principalmente pelo aumento da competição pelos sítios ativos disponíveis no adsorvente. 


\subsection{Modelagem Cinética}

Os dados obtidos nos ensaios de remoção em sistema estático foram submetidos à modelagem matemática dos modelos cinéticos expostos no Item 2.3. A título de ilustração, são expostos os ajustes dos modelos para a Solução C ( $2 \mathrm{mmol} / \mathrm{L} \mathrm{Ag} \mathrm{e}$ $2 \mathrm{mmol} / \mathrm{L} \mathrm{Cu}$ ), seguidos dos parâmetros obtidos também para as demais Soluções.

A Figura 2 apresenta o ajuste dos modelos de pseudoprimeira e pseudossegunda ordem.

Figura 2 - Ajuste das curvas cinéticas para os modelos de pseudoprimeira e pseudossegunda ordem da Solução C

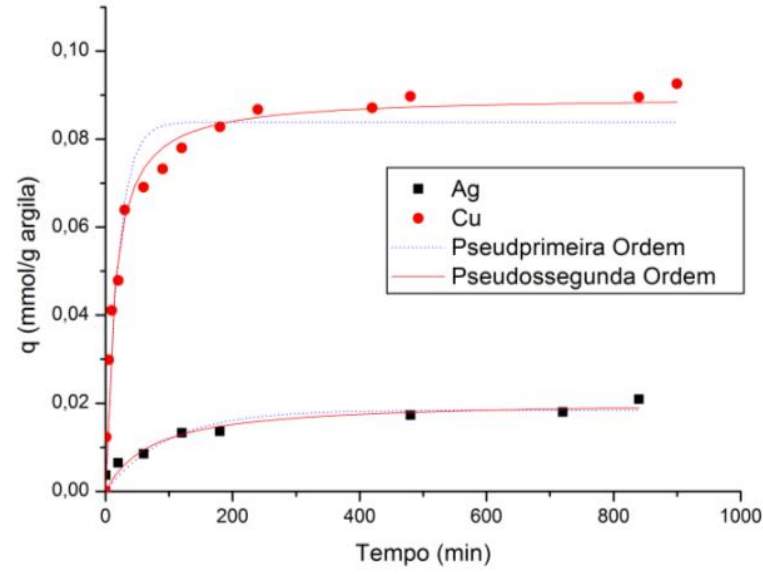

Na Figura 3 está exposto o ajuste do modelo de difusão intrapartícula.

Figura 3 - Ajuste das curvas cinéticas para o modelo de difusão intrapartícula da Solução C

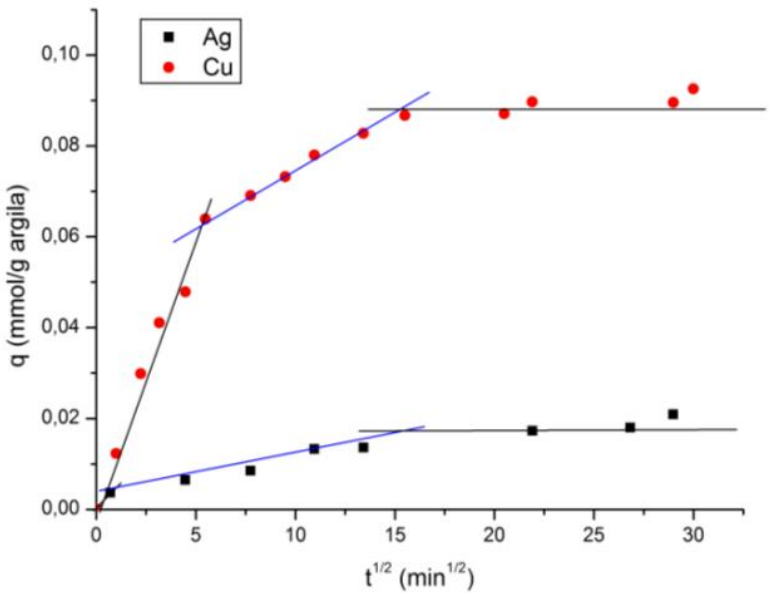

Pode-se observar nesse ajuste a existência da etapa de difusão intrapartícula para os dois metais, representada pelas retas azuis no gráfico de $\mathrm{q}$ vs. $\mathrm{t}^{1 / 2}$. Os dados dessa etapa foram ajustados linearmente para obtenção dos parâmetros do modelo, $\mathrm{k}_{\mathrm{i}}$ e c.

O último modelo, de Boyd, está apresentado nas Figuras 4 (a) e (b) para a prata e o cobre, respectivamente, como um gráfico de $B_{t}$ vs. t. Com ele, pode-se determinar a etapa limitante ao processo.

Figura 4 - Ajuste das curvas cinéticas para o modelo de Boyd da Solução C para (a) prata e (b) cobre
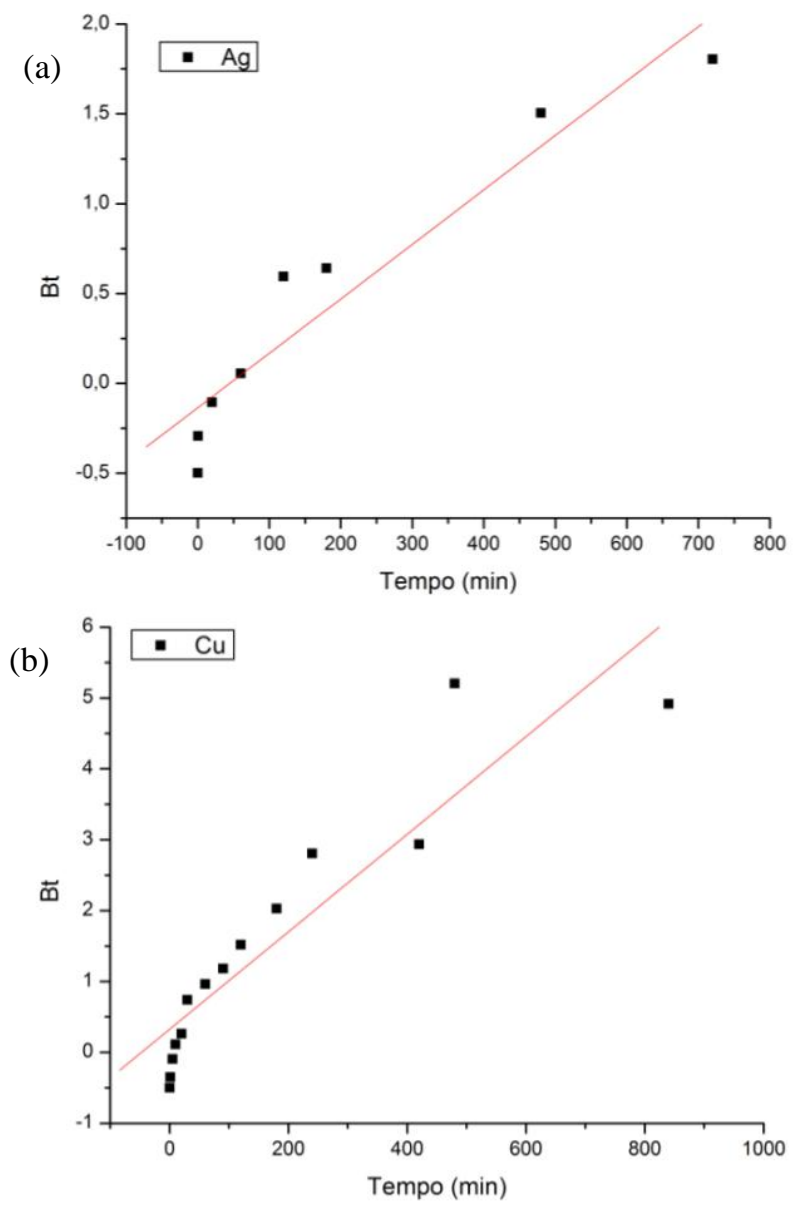

Das Figuras 4 (a) e (b), observa-se que nenhum dos ajustes lineares passa pela origem, indicando que o transporte externo controla a adsorção (BOYD, 1947). O mesmo foi observado para as Soluções A e B. 
Para cada um dos modelos analisados, foram determinados seus parâmetros e constantes, bem como o coeficiente de determinação $\mathrm{R}^{2}$ e o Desvio Médio Relativo (DMR), para avaliar a adequação de cada um aos dados experimentais.

A Tabela 2 apresenta esses resultados, sendo na primeira linha $\left(\mathrm{q}_{\mathrm{e}}\right)$ os valores experimentais observados para a quantidade adsorvida no equilíbrio.

Tabela 2 - Parâmetros obtidos para ajustes dos modelos cinéticos

\begin{tabular}{|c|c|c|c|c|c|c|c|}
\hline \multirow{3}{*}{ Modelo } & \multirow{3}{*}{ Parâmetro } & \multicolumn{6}{|c|}{ Solução } \\
\hline & & \multicolumn{2}{|c|}{$\mathrm{A}$} & \multicolumn{2}{|c|}{ B } & \multicolumn{2}{|c|}{$\mathrm{C}$} \\
\hline & & $\mathrm{Ag}$ & $\mathrm{Cu}$ & $\mathrm{Ag}$ & $\mathrm{Cu}$ & $\mathrm{Ag}$ & $\mathrm{Cu}$ \\
\hline Experimental & $\mathrm{q}_{\mathrm{e}}(\mathrm{mmol} / \mathrm{g})$ & 0,015 & 0,040 & 0,040 & 0,082 & 0,020 & 0,090 \\
\hline \multirow{4}{*}{$\begin{array}{l}\text { Pseudoprimeira } \\
\text { ordem }\end{array}$} & $\mathrm{q}_{\mathrm{e}}(\mathrm{mmol} / \mathrm{g})$ & 0,014 & 0,036 & 0,035 & 0,077 & 0,018 & 0,084 \\
\hline & $\mathrm{k}_{1}\left(\min ^{-1}\right)$ & 0,074 & 0,109 & 1,121 & 0,081 & 0,010 & 0,051 \\
\hline & $\mathrm{R}^{2}$ & 0,950 & 0,905 & 0,795 & 0,682 & 0,916 & 0,945 \\
\hline & $\operatorname{DMR}(\%)$ & 19,46 & 17,06 & 15,60 & 21,74 & 22,62 & 14,51 \\
\hline \multirow{4}{*}{$\begin{array}{l}\text { Pseudossegunda } \\
\text { ordem }\end{array}$} & $\mathrm{q}_{\mathrm{e}}(\mathrm{mmol} / \mathrm{g})$ & 0,014 & 0,039 & 0,037 & 0,078 & 0,021 & 0,090 \\
\hline & $\frac{\mathrm{k}_{2}}{(\mathrm{~g} / \mathrm{mmolmin})}$ & 7,105 & 2,900 & 34,80 & 2,549 & 0,681 & 0,829 \\
\hline & $\mathrm{R}^{2}$ & 0,973 & 0,954 & 0,859 & 0,769 & 0,941 & 0,984 \\
\hline & $\operatorname{DMR}(\%)$ & 13,38 & 13,93 & 12,51 & 17,98 & 20,72 & 8,43 \\
\hline \multirow{4}{*}{$\begin{array}{c}\text { Difusão } \\
\text { Intrapartícula }\end{array}$} & $\begin{array}{c}\mathrm{k}_{\mathrm{i}} \\
(\mathrm{mmol} / \mathrm{g} \cdot \mathrm{min})\end{array}$ & $3,5 \mathrm{E}-04$ & 1,7E-03 & $1,1 \mathrm{E}-03$ & $1,4 \mathrm{E}-03$ & $8,4 \mathrm{E}-04$ & 2,3E-03 \\
\hline & $\mathrm{c}(\mathrm{mmol} / \mathrm{g})$ & 0,008 & 0,016 & 0,026 & 0,056 & 0,003 & 0,051 \\
\hline & $\mathrm{R}^{2}$ & 0,902 & 0,975 & 0,979 & 0,968 & 0,982 & 0,997 \\
\hline & $\operatorname{DMR}(\%)$ & 87,06 & 4,20 & 11,47 & 21,47 & 15,52 & 42,29 \\
\hline Modelo de & $\mathrm{D}_{\mathrm{i}}\left(\mathrm{cm}^{2} / \mathrm{min}\right)$ & $9,0 \mathrm{E}-06$ & $5,7 \mathrm{E}-06$ & $3,1 \mathrm{E}-06$ & $7,1 \mathrm{E}-06$ & $2,2 \mathrm{E}-06$ & $5,1 \mathrm{E}-06$ \\
\hline Boyd & $\mathrm{R}^{2}$ & 0,958 & 0,926 & 0,883 & 0,972 & 0,954 & 0,921 \\
\hline
\end{tabular}

De forma geral, ao se observar dados de $\mathrm{R}^{2}$ pode-se concluir que os modelos tiveram um ajuste razoável aos dados experimentais, com exceção dos modelos de pseudoprimeira e pseudossegunda ordem da Solução B, com $\mathrm{R}^{\mathbf{2}}$ menor que 0,9. Os valores de DMR confirmam esse ajuste razoável, ficando na maioria dos casos próximo a $20 \%$. Isso pode ser explicado pela composição binária da solução, na qual diferentes concentrações molares podem apresentar diferentes comportamentos, em função da quantidade de íons presentes. Pode-se constatar também que, em geral, o melhor ajuste foi para o modelo de difusão intrapartícula, não sendo possível, entretanto, compará-lo aos demais, já que abrange apenas uma parte dos dados, referente a essa etapa.
Os modelos de pseudoprimeira e pseudossegunda ordem fornecem parâmetros que devem ser analisados, para avaliar os possíveis mecanismos envolvidos. $\mathrm{O}$ melhor ajuste foi obtido no modelo de pseudossegunda ordem, o que pode ser explicado pelo fato de o de pseudoprimeira ordem não descrever adequadamente o número de sítios ativos disponíveis. Observando-se os valores de $\mathrm{q}_{\mathrm{e}}$ para ambos os modelos, observa-se que para o cobre ele aumenta com o aumento da concentração e, para a prata, o maior valor é obtido para a concentração intermediária, o que já foi explicado como influência da quantidade de íons em solução. As constantes de primeira e de segunda ordem para o cobre apresentaram um decréscimo com o aumento da 
concentração, e no caso da prata, foram maiores para a solução de concentração intermediária, e menores para a solução de maior concentração. As menores constantes de velocidade obtidas para os dois modelos e para os dois metais na Solução C indicam menor velocidade de adsorção, o que explica o observado nesse caso, de redução da adsorção, comparada aos demais.

Avaliando-se o modelo de difusão intrapartícula, com os melhores valores de $\mathrm{R}^{2}$, não é possível observar influência das concentrações iniciais sobre a constante ki e nem sobre a grandeza $\mathrm{c}$, relacionada à espessura da camada limite.

Do modelo de Boyd, foi obtido o coeficiente de difusão efetiva (Di), independente da concentração para o cobre, mas, para a prata, apresenta o menor valor, quanto mais elevada for a concentração inicial do metal.

A menor adsorção da prata, comparada ao cobre, pode ser entendida pelo $\mathrm{pH}$ do sistema experimental. Hefne et al. (2010) estudaram o efeito do $\mathrm{pH}$ sobre a adsorção de prata em argila bentonítica natural e verificaram que $\mathrm{q}_{\mathrm{e}}$ aumenta lentamente com a concentração do íon metálico em solução, até um limite de $\mathrm{pH}=6$, em solução com concentração de prata de $1000 \mathrm{ppm}$. Sari e Tüzen (2013) investigaram o efeito do $\mathrm{pH}$ da adsorção da prata sobre vermiculita modificada com óxido de manganês e observaram um pico de adsorção em $\mathrm{pH}=4,0$ com valores reduzindo para $\mathrm{pH}$ menor ou maior que 4,0. Com isso, concluiu-se que a adsorção da prata é fortemente influenciada pelo $\mathrm{pH}$ do sistema e variações de $\mathrm{pH}$ durante o experimento podem ter causado sua menor adsorção, uma vez que o cuidado tomado foi de se manter o $\mathrm{pH}$ abaixo de 5,0 e este variou naturalmente numa faixa de 4,0 a 5,0 devido a interações dos metais com a argila, dispensando a necessidade de adição de ácido ou base para controlar essa variável.
O cobre foi estudado por Bennour (2012) que verificou que sua adsorção aumenta com o $\mathrm{pH}$, atingindo seu máximo em $\mathrm{pH}=5,0$. Isso ocorreu porque acima desse valor de $\mathrm{pH}$, o cobre precipita, não mais possibilitando a adsorção.

Outra explicação para a menor adsorção da prata está na carga superficial da argila. Almeida Neto (2011) determinou o pH de carga superficial nula da argila Verde-lodo como 5,32. Considerando-se esse valor, quando se trabalha com $\mathrm{pH}$ do sistema abaixo de 5,3, tem-se um sólido carregado positivamente, e que, portanto, tem a tendência de repelir íons de carga positiva. A maior adsorção do cobre em relação à prata pode ser explicada por algumas de suas propriedades físicas, sendo a primeira delas o raio iônico.

O cobre tem raio iônico de Pauling de $0,72 \AA$ e a prata tem raio iônico de Pauling de $1,26 \AA$ (NIGHTINGALE, 1959). O elemento com menor raio iônico é mais fortemente retido ao adsorvente. Outra propriedade capaz de explicar a tendência obtida é a relação carga/raio iônico, no valor de 2,78 para o cobre e 0,79 para a prata. Um valor baixo para essa relação indica que os íons permanecem móveis em sistema aquático, enquanto valores elevados indicam tendência em ser fortemente adsorvidos (TAVARES, 2013).

A grandeza eletronegatividade não é capaz de explicar o ocorrido, uma vez que seus valores são bastante próximos (1,90 para o cobre e 1,93 para a prata (KINRAIDE e YERMIYAHU, 2007)) e, como o do cobre é menor, deveria ser menos adsorvido do que a prata. Essa diferença pode ser explicada pela constante de hidrólise $\left(10^{-7,5}\right.$ para o cobre e $10^{-12}$ para a prata para a primeira constante de hidrólise (KINRAIDE e YERMIYAHU, 2007)) Quanto maior a facilidade de hidrólise, maior a tendência do cátion metálico em ser adsorvido especificamente (MOREIRA, 2004). 


\section{CONCLUSÃO}

Através do estudo em sistema estático para remoção de prata e cobre em solução binária sobre argila bentonítica Verde-lodo, esta se mostrou bastante eficiente na remoção. Foi observada uma maior adsorção de cobre, na presença de prata, que teve sua quantidade adsorvida reduzida.

A quantidade adsorvida mostrou-se dependente da concentração, reduzindo para elevadas quantidades de íon em solução, evidenciando a competição prejudicial pelos sítios ativos disponíveis.

Os modelos cinéticos apresentaram um ajuste razoável aos dados experimentais, sendo o de difusão intrapartícula o que apresentou o melhor ajuste.

A maior adsorção do cobre pôde ser explicada devido a certas propriedades física dos íons metálicos, bem como a influência do pH sobre a adsorção.

\section{NOMENCLATURA}

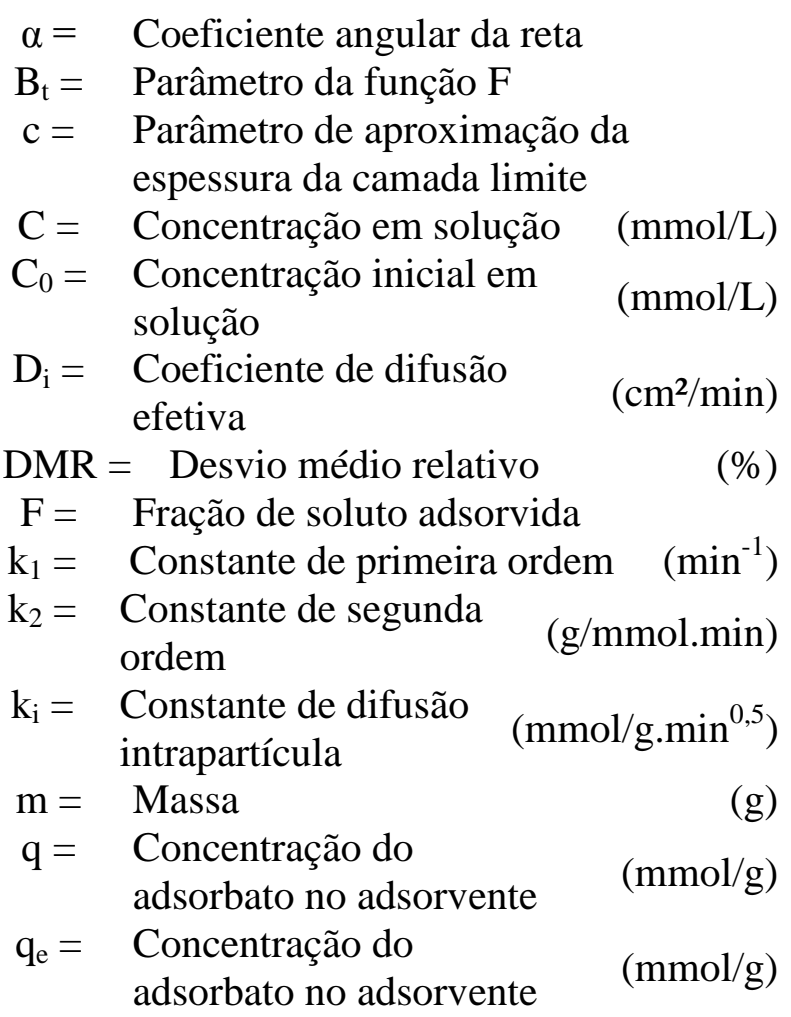

$\begin{array}{clr} & \text { no equilíbrio } & \\ \mathrm{r}= & \text { Raio médio } & (\mathrm{cm}) \\ \mathrm{R}^{2}= & \text { Coeficiente de determinação } & \\ \mathrm{t}= & \text { Tempo } & \text { (min) } \\ \mathrm{V}= & \text { Volume } & \text { (L) }\end{array}$

\section{REFERÊNCIAS}

AGUIAR, M.R.M.P., NOVAES, A.C. Remoção de metais pesados de efluentes industriais por aluminossilicatos. Química Nova, v. 25, n. 6B, p. 1145 - 1154, 2002.

ALLOWAY, B. J. Heavy metals in soil. United Kingdom: Springer, 2010.

ALMEIDA NETO, A. F., Caracterização e avaliação de argilas como adsorventes na remoção e eluição de íons cobre e mercúrio em diferentes sistemas. Tese (Doutorado em Engenharia Química) - Universidade Estadual de Campinas, Campinas, 2011.

BARAKAT, M. A. New trends in removing heavy metals from industrial wastewater. Arabian Journal of Chemistry, v. 4, p. 361377, 2011.

BARROS, A. M. Bioadsorção e dessorção dos íons $\mathrm{Cd}^{2+}, \mathrm{Cu}^{2+}, \mathrm{Ni}^{2+}, \mathrm{Pb}^{2+}$ e $\mathrm{Zn}^{2+}$ pela macrófita aquática azolla pinnata. Dissertação (Mestrado em Engenharia Química) - Universidade Estadual de Campinas, Campinas, 2012.

BENNOUR, H.A.M. Influence of $\mathrm{pH}$ and Ionic Strength on the Adsorption of Copper and Zinc in Bentonite Clay. Chemical Science Transactions, 1(2), p. $371-381$, 2012.

BOYD, G. E., ADAMSON, A. E., MEYERS, L. S. The exchange of adsorption ions from aqueous solutions by organic zeolites II. 
Kinetics. Journal of American Chemical Society, v. 69, p. 2836-2848, 1947.

CANTUARIA, M. L. Remoção de prata iônica monovalente por adsorção em argila bentonítica. Dissertação (Mestrado em Engenharia Química) - Universidade Estadual de Campinas, Campinas, 2014.

CHEN, J. P., WU, S., CHONG, K-H. Surface modification of a granular activated carbon by citric acid for enhancement of copper adsorption. Carbon, v. 41, p. 1979-1986, 2003.

DING, L., DENG, H., WU, C., HAN, Z. Affecting factors, equilibrium, kinetics and thermodynamics of bromide removal from aqueous solutions by MIEX resin. Chemical Engineering Journal, 181-182, p. 360 - 370, 2012.

GUPTA, V.K., ALI, I. Removal of ddd and dde from wastewaterusing bagasse fly ash, a sugar industry waste. Water Research, v. 35, p. $33-40,2001$.

HEFNE, J.A., MEKHEMER, W.K., ALANDIS, N.M., ALDAYEL, O.A., ALAJYAN, T. Removal of Silver (I) from Aqueous Solutions by Natural Bentonite. Science, v. 22, n.1, p. 155-176, 2010.

HO, Y.S., McKAY, G. A comparison of chemisorption kinetic models applied to pollutant removal on various sorbents. Trans IChemE, vol. 76, parte B, 1998.

HO, Y.S. Citation review of Lagergren kinetic rate equationon adsorption reactions. Scientometrics, v. 59, p. 171 - 177, 2004.

HOMEM, E.M. Remoção de Chumbo, Níquel e Zinco em Zeólita Utilizando Sistema de Leito Fluidizado. Dissertação (Mestrado em Engenharia Química) -
Universidade Estadual de Campinas, Campinas, 2001.

KINRAIDE, T.B., YERMIYAHU, U. A scale of metal ion binding strengths correlating with ionic charge, Pauling electronegativity, toxicity, and other physiological effects. Journal of Inorganic Biochemistry, v. 101, p. $1201-1213,2007$.

LAGERGREN,S. Zur theorie der sogenannten adsorption gelöster stoffe, Kungliga Svenska Vetenskapsakademiens. Handlingar, v.24, p. 1 -39, 1898.

MOREIRA, C.S. Adsorção competitiva de cádmio, cobre, níquel e zinco em solos. Dissertação (Mestrado em Agronomia) Universidade de São Paulo, Piracicaba, 2004.

MOREIRA, G.H., CARVALHO, L.M. Galvanoplastia. São Paulo:FATEC, 2012.

NIGHTINGALE, E.R. Phenomenological theory of ion solvation. Effective radii of hydrated ions. Journal Physical Chemistry, v. 63 , p. 1381 - 1387, 1959.

SARI, A., TÜZEN, M., Adsorption of silver from aqueous solution onto raw vermiculite and manganese oxide-modified vermiculite. Microporous and Mesoporous Materials, $n$. 170, p. 155 - 163, 2013.

SONG, X., GUNAWAN, P., JIANG, R., LEONG, S.S.J., WANG, K., XU, R. Surface activated carbon nanospheres for fast adsorption of silver ions from aqueous solutions. Journal of Hazardous Materials. n. 194, p. $162-168,2011$.

TAVARES, S.R.L. Remediação de solos e águas contaminadas por metais pesados: conceitos básicos e fundamentos. Rio de Janeiro, 2013. 
WEBER, W. J.; MORRIS, J. C. Kinetics of adsorption on carbon from solution. Journal of the Sanitary Engineering Division, v. 89, p. 31-60, 1963.

YUN, Y-S., PARK, D., PARK, J.M., VOLESKY, B., Biosorption of trivalent chromium on the brow seaweed biomass. Environmental Science Technology, v. 35, p. 4353-4358, 2001.

\section{AGRADECIMENTOS}

Os autores agradecem à FAPESP (Processos n ${ }^{\circ}$ 2013/00732-1 e 2013/25212-0) pelo auxílio financeiro e bolsa concedida. 